Review Article

\title{
The Efficacy and Safety of Simple-Needling Therapy for Treating Ankylosing Spondylitis: A Systematic Review and Meta-Analysis of Randomized Controlled Trials
}

\author{
Yichen Xuan $\mathbb{D}^{1},{ }^{1}$ Hui Huang $\mathbb{D}^{2},{ }^{2}$ Yiyong Huang, ${ }^{1}$ Duanyong Liu, ${ }^{1}$ Xiuwu Hu, ${ }^{2}$ \\ and Lele Geng ${ }^{2}$ \\ ${ }^{1}$ Science and Technology College of Jiangxi University of Traditional Chinese Medicine, Nanchang, China \\ ${ }^{2}$ Department of Acupuncture, Nanchang Hongdu Hospital of Traditional Chinese Medicine, Nanchang, China \\ Correspondence should be addressed to Hui Huang; 565024534@qq.com
}

Received 8 September 2019; Revised 22 February 2020; Accepted 14 May 2020; Published 13 June 2020

Academic Editor: Ho Lin

Copyright (C) 2020 Yichen Xuan et al. This is an open access article distributed under the Creative Commons Attribution License, which permits unrestricted use, distribution, and reproduction in any medium, provided the original work is properly cited.

\begin{abstract}
Background. Clinical investigators have found that the use of needling in the treatment of ankylosing spondylitis (AS) has a good clinical application prospect in recent years. However, these studies were insufficient to provide evidence for the efficacy and safety of simple-needling for AS. So, we performed a systematic review and meta-analysis to evaluate the efficacy and safety of simple-needling for treating AS. Methods. We searched the PubMed, Cochrane Library, Embase, China National Knowledge Infrastructure (CNKI), Chinese Biomedical Literature Database (CBM), Wangfang database (Wanfang), Chinese Science and Technology Periodical Database (VIP), and any other gray literature sources for randomized controlled trials (RCTs) that used simple-needling to treat AS before June 2019 with the language restriction of Chinese and English. Researchers evaluated the retrieved literature studies and extracted valid data according to relevant requirements and used RevMan5.3 software for meta-analysis. Results. A total of 10 studies were included, all of which were Chinese literature studies, involving 729 patients. Compared with the control groups, simpleneedling groups had a better effect on the clinical effective rate ( $\mathrm{RR}=1.20,95 \% \mathrm{CI}(1.11,1.29), P<0.00001)$, TCM syndrome score $(\mathrm{MD}=-5.26,95 \% \mathrm{CI}(-5.99,-4.53), P<0.00001)$, symptom score $(\mathrm{MD}=-8.08,95 \% \mathrm{CI}(-10.18,-5.97), P<0.00001)$, and Schober test outcome $(\mathrm{MD}=0.39,95 \% \mathrm{CI}(0.15,0.64), P=0.002)$. Sensibility analysis was based on the leave-one-out cross-validation procedure, and the results showed no significant changes. Most studies did not describe adverse reactions. The funnel plot suggested publication bias on clinical effectiveness. Conclusions. This systematic review and meta-analysis demonstrated that simple-needling was effective as an intervention for AS. However, due to the low quality of the methodology of included studies, the designs of clinical trials were not rigorously standardized. Therefore, it is necessary to carry out multiquality RCTs for verification.
\end{abstract}

\section{Introduction}

Ankylosing spondylitis (AS) is the main form of chronic inflammatory arthritis affecting the axial skeleton and is characterized by excess spinal bone formation, inflammatory back pain, radiographic sacroiliitis, and a high prevalence of HLA-B27 $[1,2]$. In 2014, the estimated number of patients with AS was from 1.30 to 1.56 million in Europe and from 4.63 to 4.98 million in Asia [3]. In 2017, a study enrolled 1251 AS patients from China. The results showed that the mean age of onset and diagnosis was 29.2 and 33.5 years, and the ratio of male to female was $2.7: 1$ [4]. A recent study reported that the risk of cardiovascular events in AS patients increased by $30-50 \%$ compared with the general population [5]. In addition, patients with AS had increased risks of multiple diseases such as chronic obstructive pulmonary disease, asthma, type 2 diabetes, stroke, cancer, and depression [6-13]. The occurrence of these comorbidities made us have to pay attention to the treatment of AS. The American College of Rheumatology and Spondylitis Association of America recommended that nonsteroidal antiinflammatory drugs (NSAIDs) and tumor necrosis factor inhibitors (TNFi) remain the primary classes of medications for the treatment of AS in 2019 [14]. Common adverse 
reactions to medications such as abdominal pain, loss of appetite, upper gastrointestinal bleeding, and other important factors restrict the treatment of some patients. Furthermore, the cost of medicine must be considered for patients. For example, TNFi therapy can effectively improve joint mobility in patients with AS [15] and delay patients' spine imaging changes [16]. However, the cost of medicine has brought a large financial burden to AS patients and their families [17]. Needling, as a traditional Chinese therapy, is a popular complementary and alternative therapy in the world $[18,19]$. Due to limited evidence to support the effectiveness of needling for AS, needling is not a standard treatment for AS. But as an unconventional therapy, needling is the choice of many patients [20]. In order to obtain more effective treatment for patients, needling combined with other therapies had been commonly used in China to treat AS, such as needling combined with moxibustion, traditional Chinese medicine (TCM), and sulfasalazine [21-23]. The efficacy and superiority of needling combined with other therapies for AS patients had been evaluated and verified in the meta-analysis [24-27]. Considering that the therapeutic effects of simple-needling therapy could not be distinguished. Therefore, to evaluate the efficacy and safety of simple-needling therapy, we comprehensively collected RCTs of simple-needling therapy in the treatment of AS and conducted a systematic review and meta-analysis.

\section{Data and Method}

\subsection{Eligibility Criteria}

2.1.1. Types of Studies. The included studies were RCTs in Chinese and English.

2.1.2. Types of Participants. All participants were clearly diagnosed as AS, and there were no restrictions on the gender, age, and duration of the study subjects.

2.1.3. Types of Interventions. Interventions in the experimental groups included the use of needling therapy alone. Trials that used a combination of other interventions were excluded, such as other Chinese medicines, Western medicine, moxibustion, and Tuina. The interventions in the control groups were nonneedling treatments.

2.1.4. Types of Outcome Measures. Included trials must report outcome indicators in at least one of the following forms: (1) clinical effective rate, the proportion of patients including improvement, effectiveness, and cure, according to the efficacy standards of each clinical study; (2) TCM syndrome score; (3) symptom score; (4) Schober test outcome; and (5) adverse reactions.

2.2. Exclusion Criteria. Exclusion criteria were as follows: (1) nonclinical RCTs, such as animal experiments, molecular experiments, clinical experience, and research progress; (2) repeated publication and trials with the same original data;
(3) inability to obtain the original text, and data that could not be extracted from the tests; (4) research designs that were unreasonable; and (5) the intervention measures of the experimental groups that were moxibustion or acupotomy.

2.3. Search Strategy. We searched the following electronic databases by computer: PubMed, the Cochrane Library, Embase, China National Knowledge Infrastructure (CNKI), Chinese Biomedical Literature Database (CBM), Wanfang database (WF), and Chinese Science and Technology Periodical Database (VIP). We searched from inception to June 2019. The search was carried out by means of a combination of Medical Subject Heading (MeSH) terms and free words. The search terms were ankylosing spondylitis, acupuncture, acupuncture therapy, needle, needling, and randomized. We also retrieved any other gray literature sources.

2.4. Screening of Literature Studies. All the searched literature titles were retrieved into EndnoteX9, manual auxiliary operation combined with EndnoteX9 to review the literature, and eliminated the duplicate publication of literature. Two researchers separately read the titles and abstracts and deleted the documents that were obviously unqualified according to the inclusion and exclusion criteria. The rest of the literature were read in full text and finally confirmed the included literature. If there were different opinions between the two researchers in the process of literature screening, they would need to be resolved through negotiation. If the outcome of the consultation was not reached, it would be determined by the third researcher.

2.5. Data Extraction. The included literature studies were read independently by two researchers who extracted the data. The extraction included the authors' name, year of publication, literature sources, sample size, baselines, research designs, diagnostic criteria, random methods, distribution concealment, blind implementation, missing or shedding reports, interventions, courses of treatment, efficacy criteria, efficacy indicators, and adverse events. If the information about the original studies was missing or unclear, we would contact the lead author by e-mail or phone as far as possible.

2.6. Quality Assessment. Based on the bias risk assessment method in the Cochrane handbook [28], two researchers independently analyzed the quality of the included literature. If there were differences, they would defer to third-party opinions and reach an agreement. The evaluation included (1) random sequence generation, (2) allocation concealment, (3) blinding implementation, (4) incomplete outcome data, (5) selective reporting, and (6) other sources of bias.

2.7. Statistical Analysis. RevMan5.3 software was used for data analysis. The results were analyzed to evaluate the difference between needling therapy and control groups. The mean difference (MD) was used to represent the 
measurement data, and the relative risk (RR) was used to represent the counting data. Each effect was expressed by a 95\% confidence interval (CI), and the difference was statistically significant $(P<0.05)$. Heterogeneity within RCTs was examined based on the $I^{2}$ test. $I^{2} \leq 50 \%$ was considered that there was low heterogeneity between the studies. Metaanalysis was carried out by using the fixed effect model. $50 \%<I^{2}<75 \%$ was considered that there was moderate heterogeneity, and $I^{2} \geq 75 \%$ was considered that there was significant heterogeneity. Meta-analysis was carried out by using a random effect model, and its heterogeneity sources were analyzed. If the data could not be merged, the descriptive analysis would be used.

2.8. Sensitivity Analysis. Sensitivity analysis was based on the leave-one-out cross-validation procedure to clarify the stability and reliability of the results.

2.9. Publication of Bias Risk Assessment. If the number of studies for pooling was not less than 8 , publication bias was assessed using a funnel plot.

\section{Result}

3.1. Screened Results of Literature Studies. A total of 1289 articles, 43 English literature studies and 1246 Chinese literature studies, were searched. After screening, a total of 10 studies [29-38] were included, all of which were Chinese literature studies (Figure 1).

3.2. Characteristics of Included Literature Studies. A total of 729 patients, 377 patients in the experimental groups and 352 patients in the control groups, were enrolled in 10 RCTs. The intervention measures of the experimental groups were simple-needling, while those of the control groups were the routine treatment of Western medicine (Table 1).

3.3. Diagnostic Criteria of Included Studies. Two studies $[30,37]$ referred to Western medicine diagnostic criteria and TCM diagnostic criteria, respectively. The diagnostic criteria for Western medicine were the New York standards revised in 1984, and the diagnostic criteria for TCM were the Principles for Clinical Research of New TCM in 2002 and Wang Yongyan's Internal Medicine for TCM in 2001. The remaining eight studies $[29,31-36,38]$ referred to Western medicine diagnostic criteria: four studies [31, 32, 34, 35] were based on the New York Standard revised in 1984, one study [29] was based on the Guideline for the Diagnosis and Treatment of AS with Chinese Medical Association Rheumatology Branch in 2010, one study [36] was based on the diagnostic criteria developed by the Academic Conference on Integrative Medicine for Rheumatology in 1984, one study [38] was based on the New York Standard integrated with the Academic Conference on Integrative Medicine for Rheumatology in 1984, and one study [33] did not describe the diagnostic criteria.
3.4. Efficacy Criteria of Included Studies. Eight studies $[29,30,32-34,36-38]$ described the efficacy criteria of the RCTs: two studies $[33,34]$ referred to the Diagnostic and Efficacy Standards for TCM Diseases promulgated by the Chinese Medicine Administration in 1994, one study [29] referred to the Principles for Clinical Research of New TCM in 2002, one study [32] was based on the National Rheumatology Conference in 1998, one study [37] referred to Wang Yongyan's Internal Medicine for TCM in 2001, and three studies $[30,36,38]$ were based on self-made criteria. The remaining 2 studies $[31,35]$ did not address the efficacy criteria.

3.5. Quality Evaluation of Included Studies. The quality of the 10 studies was low. 7 studies $[29,31-35,38]$ reported the random sequence generation, and 3 studies $[30,36,37]$ had only random words. None of the 10 studies [29-38] described the implementation of allocation concealment. One study [38] described the implementation of single blindness, and the remaining 9 studies [29-37] did not describe the implementation of blinding. The final outcomes reported in the 10 studies [29-38] were consistent with the outcomes they set, so the outcome data were complete. The information for all studies was insufficient to determine whether selective reports exist and failed to obtain other sources of bias (Figures 2 and 3).

\subsection{Meta-Analysis}

3.6.1. Clinical Effective Rate. The combined results of 8 trials $[29,30,32-34,36-38]$ released the idea that simple-needling group was better than the control group statistically in the clinical effective rate $(\mathrm{RR}=1.20,95 \%$ CI $(1.11,1.29)$, $P<0.00001)$. Low heterogeneity between the studies was found $\left(I^{2}=0 \%\right)$ using the fixed-effect model (Figure 4).

3.6.2. TCM Syndrome Score. There was a significant difference in TCM syndrome score according to the combined results of 2 studies $[30,31](\mathrm{MD}=-5.26,95 \%$ CI $(-5.99$, $-4.53), P<0.00001)$. According to the heterogeneity test, there was low heterogeneity between the 2 studies $\left(I^{2}=0 \%\right)$, using the fixed-effect model. Figure 5.

3.6.3. Symptom Score. There were 3 RCTs $[35,36,38]$ for meta-analysis that compared the outcome of symptom score. The result showed a significant difference between the simple-needling group and the control group $(\mathrm{MD}=-8.08$, $95 \%$ CI $(-10.18,-5.97), P<0.00001)$. The random-effect model was used owing to their significant heterogeneity $\left(I^{2}=86 \%\right)$. We reviewed the original literature studies to infer that the sources of heterogeneity may be related to different scoring criteria or the difference between patients or different operators (Figure 6).

3.6.4. Schober Test Outcome. Two RCTs $[29,32]$ reported the Schober test outcome, and a significant difference between the two interventions was found from the combined results 


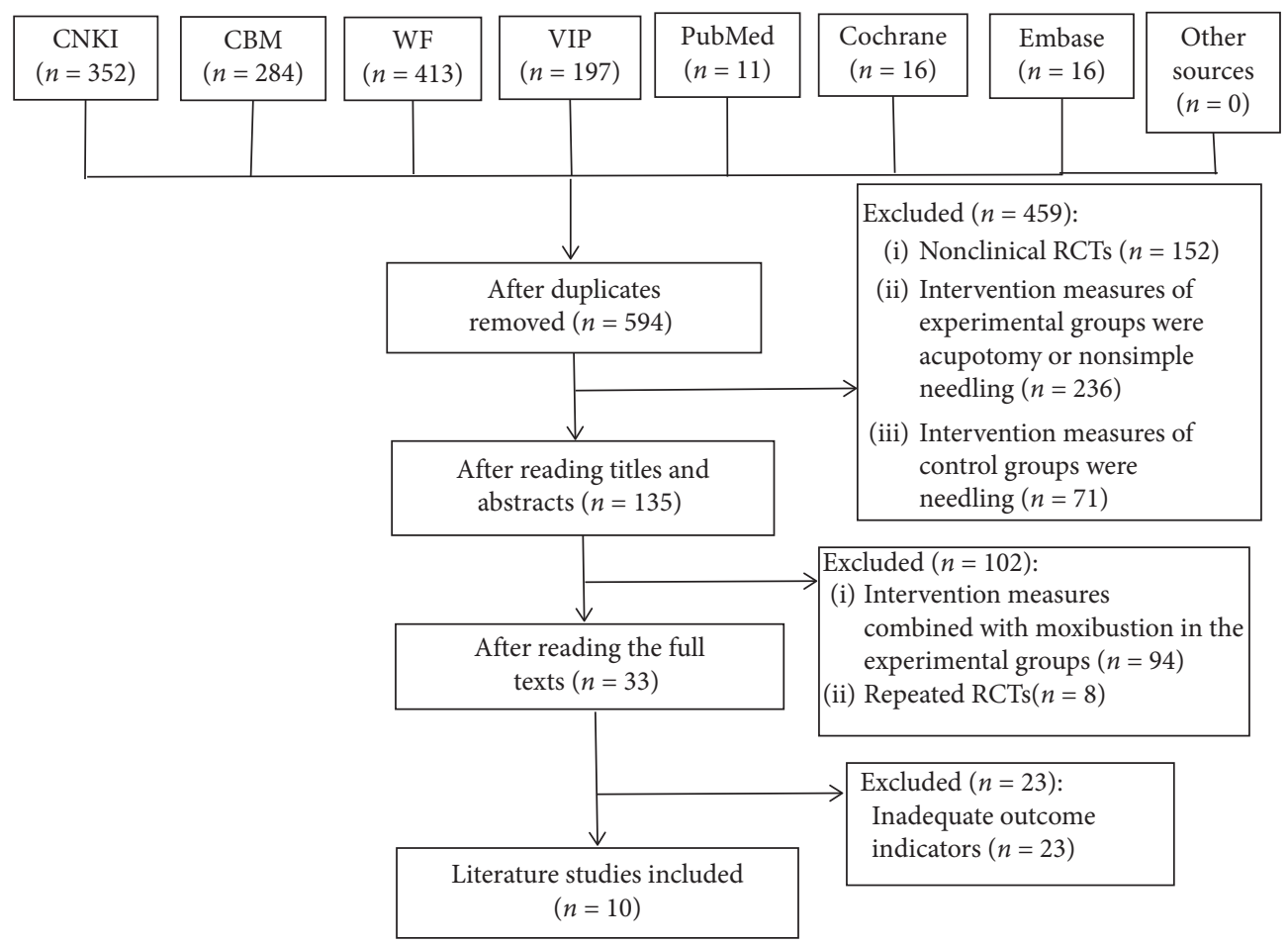

FIgURe 1: Flow diagram of screening literature studies.

indicated $(\mathrm{MD}=0.39,95 \% \mathrm{CI}(0.15,0.64), P=0.002)$. There was low heterogeneity between the 2 studies $\left(I^{2}=0 \%\right)$, using the fixed-effect model. Figure 7.

3.7. Sensibility Analysis. We conducted the sensibility analysis based on the leave-one-out cross-validation procedure, and the results showed no significant changes, indicating that the meta-analysis results were more reliable.

3.8. Safety Evaluation. Only 2 studies [31, 35] described adverse reactions, and there were no obvious adverse reactions in the experimental groups.

3.9. Publication Bias. A meta-analysis of the clinical effectiveness of the 8 studies [29, 30,32-34, 36-38] and a funnel plot showed asymmetrical and skewed distributions. This may have publication bias (Figure 8).

\section{Discussion}

4.1. Needling Treatment of the Potential of AS. AS is a chronic inflammatory autoimmune disease that mainly causes lumbosacral pain and stiffness and finally affects the entire spine [1]. At present, adverse reactions and financial burdens are caused to patients by current medication treatment to a certain extent, and needling therapy as a supplementary replacement therapy has attracted the attention of scholars because of its unique curative effects and low side effects [39]. From the perspective of modern medicine, needling therapy has an obvious analgesic effect [40] and improves the immune function of the bodies [41]. From the perspective of traditional meridian theory, it is considered that kidney deficiency is the basis of AS generation, and kidney essence can be supplemented by needling therapy [42]. Studies had shown that needling therapy could effectively alleviate spinal pain, improve spinal function, and significantly reduce the levels of TNF- $\alpha$ and IL- $1 \beta$ inflammatory factors in patients with AS [43-45].

4.2. Systematic Review and Meta-Analysis Main Findings. In this review, we identified 10 studies of simple-needling for AS, and these studies included 729 participants. All the interventions included in the studies were compared with simple-needling treatment and conventional Western medicine treatment. The results showed that the clinical effective rate of simple-needling was higher than that of Western medicine, and it was better than the intervention of control groups in improving TCM syndrome score, symptom score, and spinal function.

4.3. Systematic Review and Meta-Analysis Advantages. The meta-analysis was proposed by the American educator Glass in 1976 [46]. Its essence is to qualitatively or quantitatively synthesize multiple independent studies, solve clinical divergence opinions, and enhance the reliability and objectivity of clinical application effects. Meta-analysis has been widely used by various clinical medicine majors in recent years [47]. This is the first meta-analysis and systematic review of simple-needling for the intervention of AS. The use of English and Chinese databases allowed access to publications in common languages for needling research and retrieved any other gray literature sources. Through the 


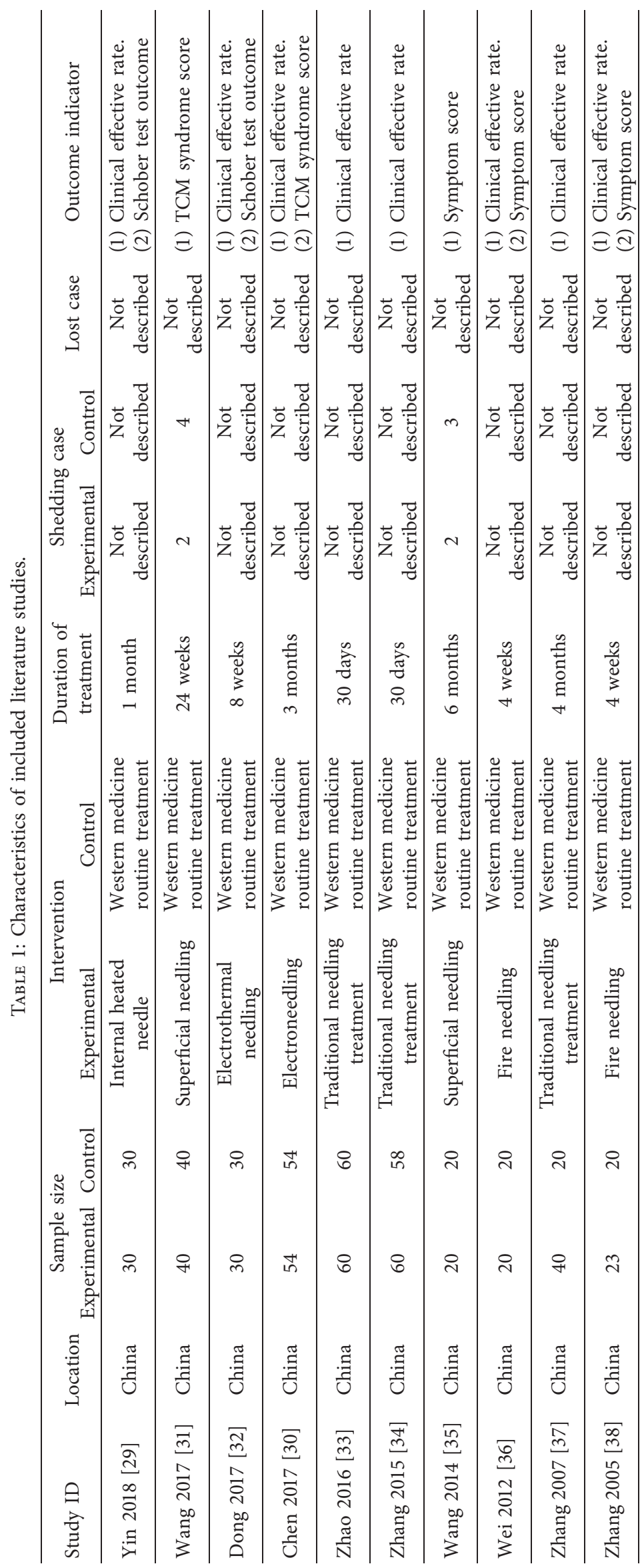




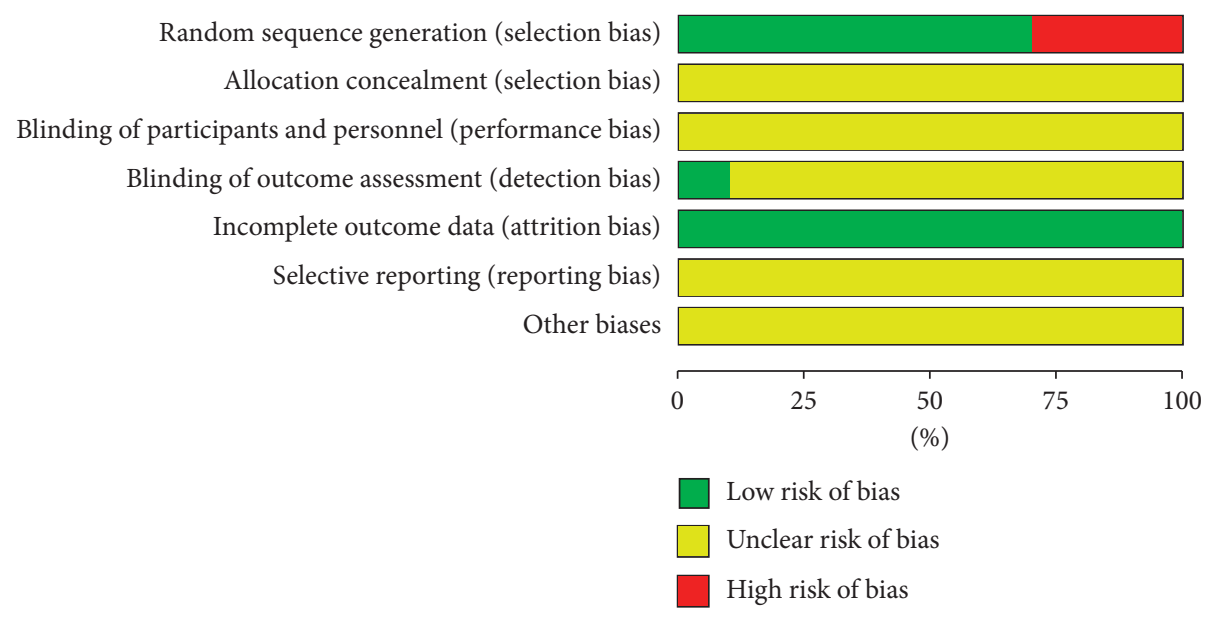

FIgURE 2: Risk of bias graph.

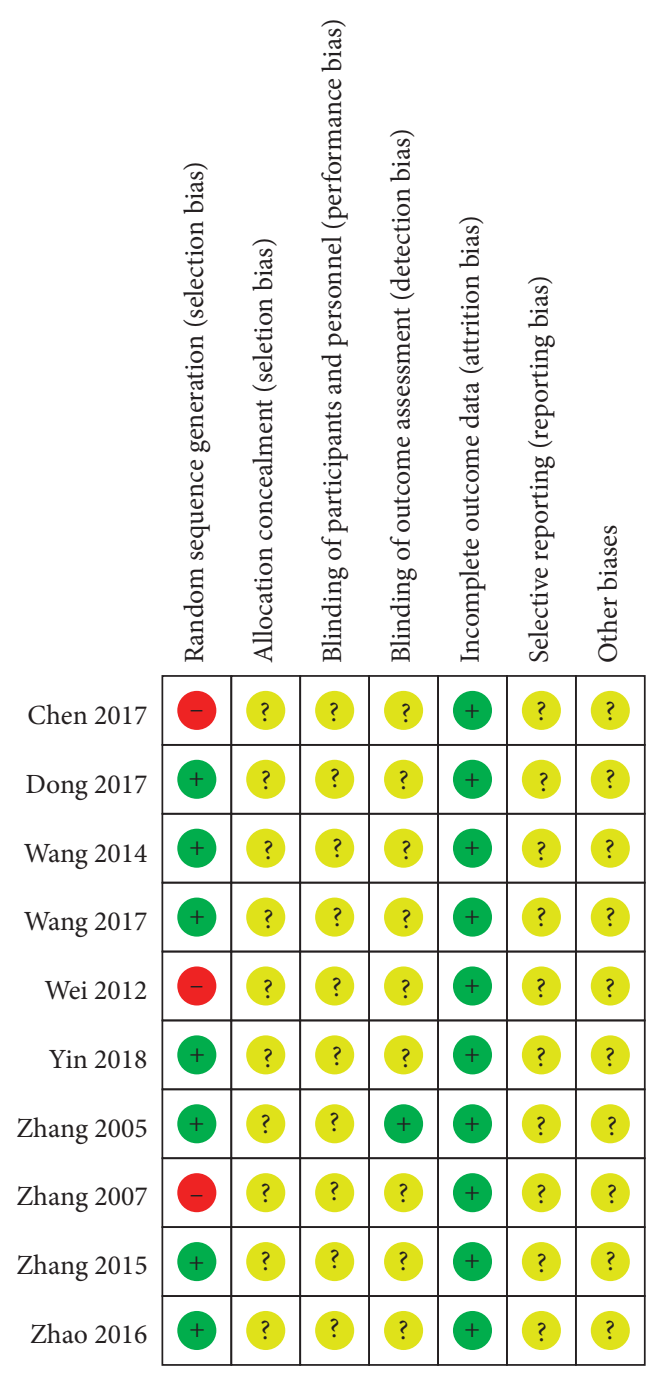

FIGURE 3: Risk of bias summary. 


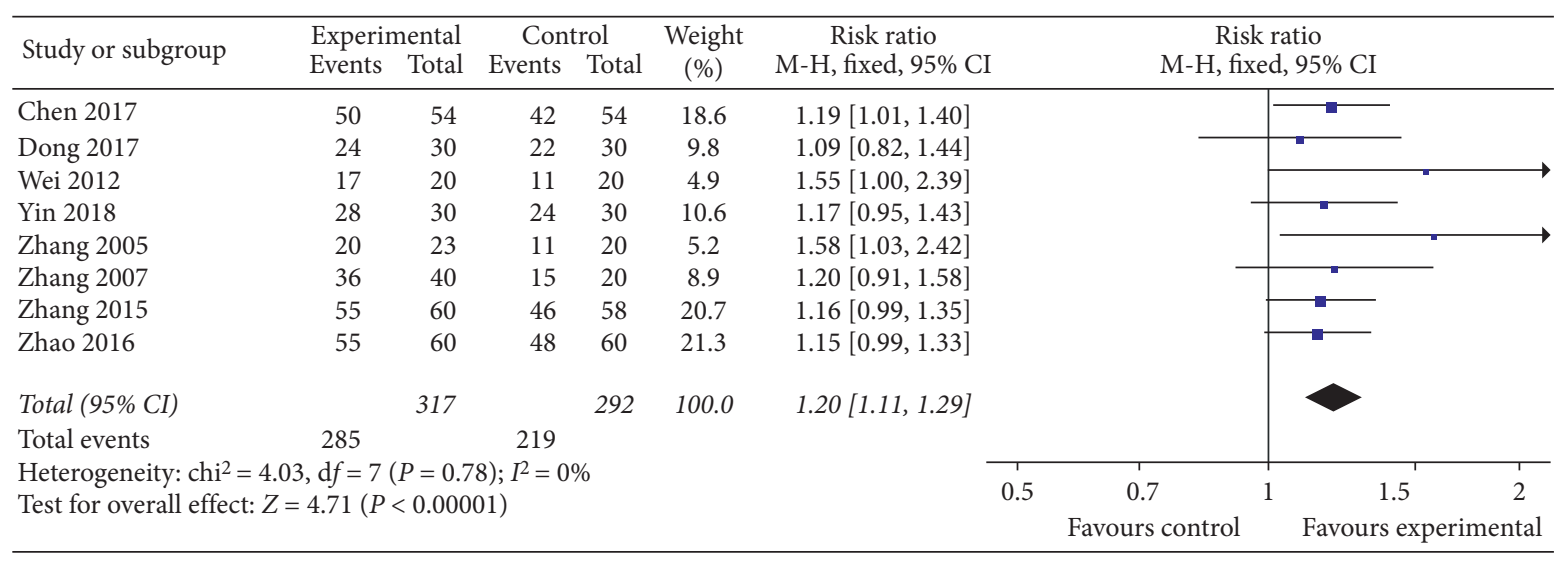

FIGURE 4: Forest plot of experimental groups versus control groups: clinical effective rate.

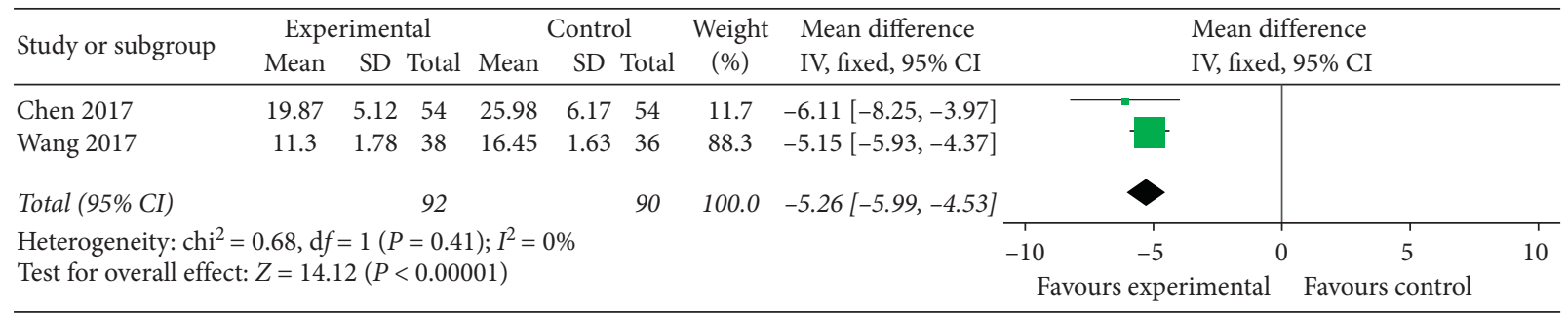

FIGURE 5: Forest plot of experimental groups versus control groups: TCM syndrome score.

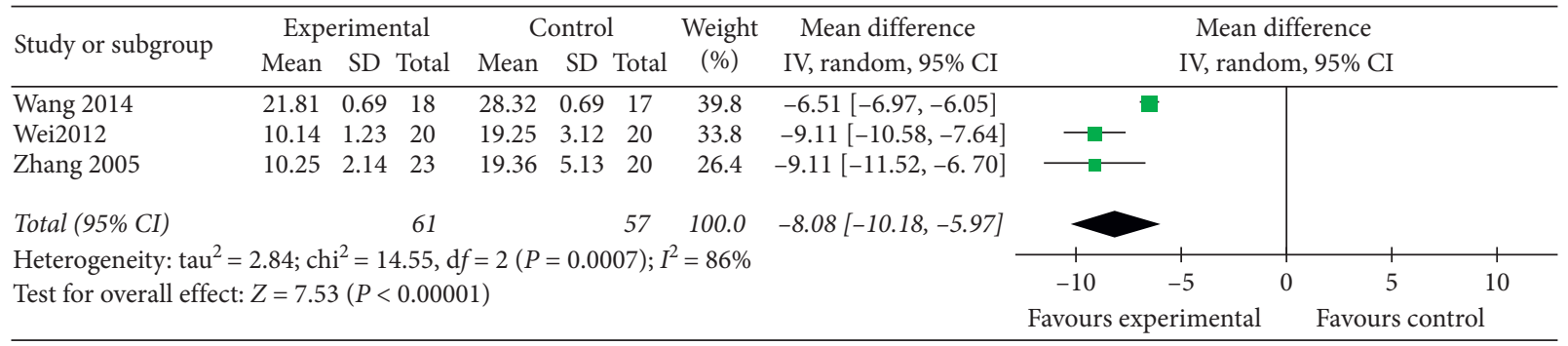

FIGURE 6: Forest plot of experimental groups versus control groups: symptom score.

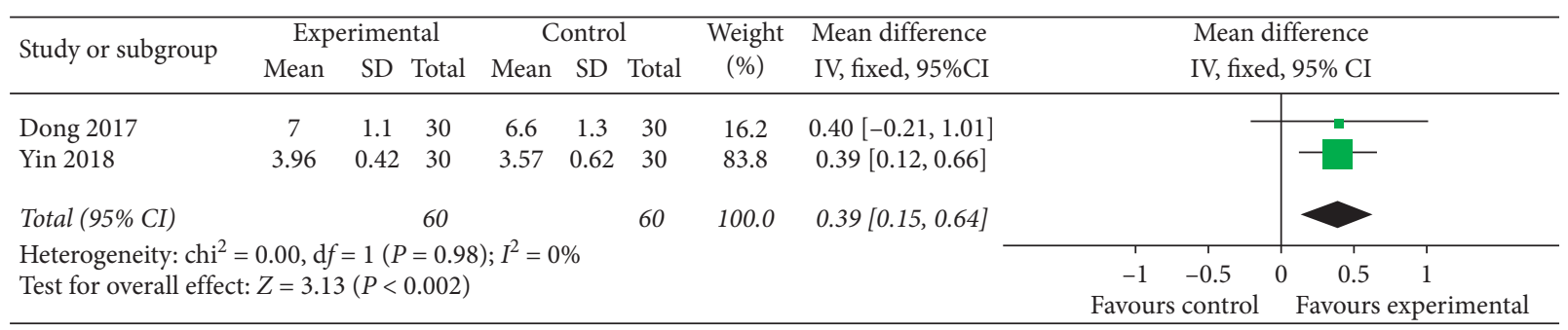

Figure 7: Forest plot of experimental groups versus control groups: Schober test outcome.

meta-analysis, we analyzed the data of the included studies to clarify that the simple-needling is effective as an intervention for AS and to provide a more reliable basis for clinical decision-making.

4.4. Systematic Review and Meta-Analysis Limitations. This systematic review and meta-analysis had several limitations. (1) Although we had retrieved a large number of
Chinese and English studies, the final included studies were published in Chinese. The patients included were all from China. (2) The sample size included in the studies was small, and most studies did not describe case shedding. Only two studies described case shedding, but there was no intentional analysis. (3) In the included studies, simple-needling therapy was used as an experimental group compared with conventional Western medicine treatment. The results of each study were positive for simple-needling treatment. We may 


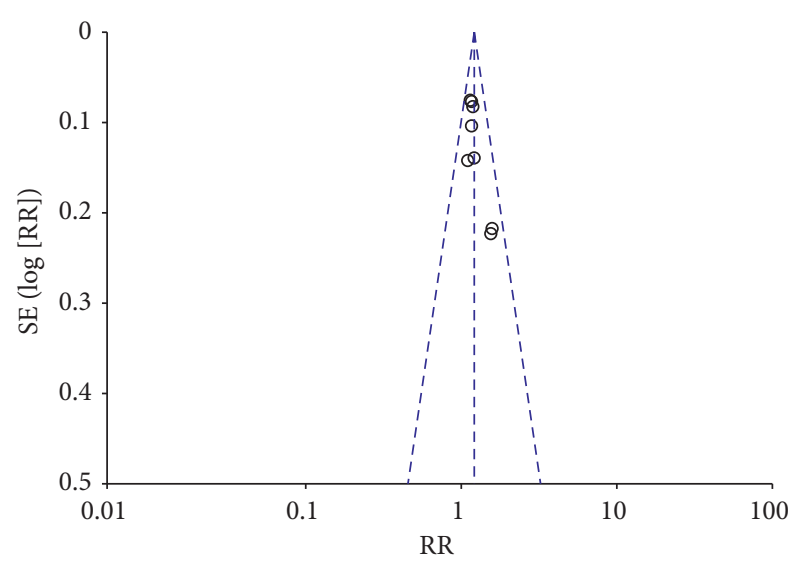

FIgURE 8: Clinical effective rate funnel plot.

miss some negative data, which could lead to an overestimate of the effectiveness of simple-needling for AS. (4) The diagnostic criteria and efficacy criteria of RCTs were inconsistent and may result in selection and measurement bias. (5) 7 studies described random methods, 3 studies had only random words, but the details of randomization procedures were often absent. None of the RCTs described the implementation of allocation concealment. One study described the implementation of single blindness, and the remaining 9 studies did not describe, which may pose risks of bias. (6) The widely validated and accepted outcome measures were not used in most of the studies we included, which may have some impact on the results of the metaanalysis. (7) All trials did not describe the follow-up; only to determine the short-term efficacy of simple-needling, its long-term efficacy was not judged. (8) Only 2 studies evaluated safety, and the rest of the studies were unable to judge their safety. (9) Although this study used a funnel plot to describe the publication bias of the clinical efficacy of simple-needling in the treatment of AS, the result may be affected by the lack of inclusion of the studies. All of the above restrictions may cause instability of the meta-analysis results.

\section{Conclusion}

Through comprehensive analysis, this study demonstrated that simple-needling was effective as an intervention for AS. However, there were few relevant studies. Including the low quality of the research methodology, the sample size was small, and the diagnostic criteria and the efficacy criteria were inconsistent, so the results were not representative. Therefore, more rigorous RCTs are needed to verify the above conclusions. In the future, such research should be carried out in accordance with the requirements of evidencebased medicine. The top-level design of research programs, random methods, allocation of hidden methods, implementation of blind methods, adverse reactions, and followup data should be valued. It is best to use the latest internationally recognized diagnostic criteria and efficacy criteria. Meanwhile, we used the outcome indicators that are widely validated and accepted at the international level and tried to compare them with other interventions, to further clarify the therapeutic advantages of simple-needling for AS and provide a reliable and objective reference for the clinical treatment of AS.

\author{
Abbreviations \\ AS: $\quad$ Ankylosing spondylitis \\ RCT: Randomized controlled trial \\ TCM: Traditional Chinese medicine \\ NSAIDs: Nonsteroidal anti-inflammatory drugs \\ TNFi: $\quad$ Tumor necrosis factor inhibitors \\ CI: $\quad$ Confidence interval \\ RR: $\quad$ Relative risk \\ MD: $\quad$ Mean difference \\ CNKI: China National Knowledge Infrastructure \\ CBM: Chinese Biomedical Literature Database \\ WF: Wangfang database \\ VIP: Chinese Science and Technology Periodical \\ Database \\ MeSH: Medical Subject Heading.
}

\section{Conflicts of Interest}

The authors declared that there were no conflicts of interest.

\section{Authors' Contributions}

Yichen Xuan and Hui Huang were responsible for the design of the research program. Yichen Xuan and Yiyong Huang searched and filtered related literature studies. Lele Geng and Xiuwu Hu evaluated the quality of the literature studies and collated and analyzed data. Yichen Xuan drafted the manuscript. Hui Huang and Duanyong Liu revised the manuscript. All authors read the manuscript and approved the final version of the manuscript for publication.

\section{Acknowledgments}

This research work was supported by the Traditional Chinese Medicine Science and Technology Programme Project of Health and Wellness Committee of Jiangxi Province (no. 2018A393).

\section{References}

[1] J. Braun and J. Sieper, "Ankylosing spondylitis," The Lancet, vol. 369, no. 9570, pp. 1379-1390, 2007.

[2] R. Wang and M. M. Ward, "Epidemiology of axial spondyloarthritis," Current Opinion in Rheumatology, vol. 30, no. 2, pp. 137-143, 2018.

[3] L. E. Dean, G. T. Jones, A. G. MacDonald, C. Downham, R. D. Sturrock, and G. J. Macfarlane, "Global prevalence of ankylosing spondylitis," Rheumatology, vol. 53, no. 4, pp. 650-657, 2014.

[4] Q. Qian, X. Xu, H. He et al., "Clinical patterns and characteristics of ankylosing spondylitis in China," Clinical Rheumatology, vol. 36, no. 7, pp. 1561-1568, 2017.

[5] K. Bengtsson, H. Forsblad-d'Elia, and E. Lie, "Are ankylosing spondylitis,psoriatic arthritis and undifferentiated spondyloarthritis associated with an increased risk of cardiovascular 
events?A prospective nationwide population-based cohort study," Arthritis Research \& Therapy, vol. 19, no. 1, p. 102, 2017.

[6] K. Sharif, A. Watad, S. Tiosano et al., "The link between COPD and ankylosing spondylitis: a population based study," European Journal of Internal Medicine, vol. 53, pp. 62-65, 2018.

[7] J. H. Kang, Y. H. Chen, and H. C. Lin, "Comorbidity profiles among patients with ankylosing spondylitis:a nationwide population-based study," Annals of the Rheumatic Diseases, vol. 69, no. 6, pp. 1165-1168, 2009.

[8] A. Bremander, I. F. Petersson, S. Bergman, and M. Englund, "Population-based estimates of common comorbidities and cardiovascular disease in ankylosing spondylitis," Arthritis Care \& Research, vol. 63, no. 4, pp. 550-556, 2011.

[9] J. J. Meesters, A. Bremander, and S. Bergman, "The risk for depression in patients with ankylosing spondylitis: a population-based cohort study," Arthritis Research \& Therapy, vol. 16, no. 5, p. 418, 2014.

[10] L.-M. Sun, C.-H. Muo, J.-A. Liang, S.-N. Chang, F.-C. Sung, and C.-H. Kao, "Increased risk of cancer for patients with ankylosing spondylitis: a nationwide population-based retrospective cohort study," Scandinavian Journal of Rheumatology, vol. 43, no. 4, pp. 301-306, 2014.

[11] H.-H. Chen, S.-Y. Yeh, H.-Y. Chen, C.-L. Lin, F.-C. Sung, and C.-H. Kao, "Ankylosing spondylitis and other inflammatory spondyloarthritis increase the risk of developing type 2 diabetes in an Asian population," Rheumatology International, vol. 34, no. 2, pp. 265-270, 2014.

[12] J. J. Keller, J.-L. Hsu, S.-M. Lin et al., "Increased risk of stroke among patients with ankylosing spondylitis: a populationbased matched-cohort study," Rheumatology International, vol. 34, no. 2, pp. 255-263, 2014.

[13] J. Rosenbaum, V. Chandran, A. Deodhar, and D. Clegg, "Management of comorbidities in ankylosing spondylitis," The American Journal of the Medical Sciences, vol. 343, no. 5, pp. 364-366, 2012.

[14] M. M. Ward, A. Deodhar, L. S. Gensler et al., "2019 update of the American College of rheumatology/spondylitis association of America/spondyloarthritis research and treatment network recommendations for the treatment of ankylosing spondylitis and nonradiographic axial spondyloarthritis," Arthritis \& Rheumatology, vol. 71, no. 10, pp. 1599-1613, 2019.

[15] D. Van der Heijde, M. Breban, D. Halter et al., "Maintenance of improvement in spinal mobility, physical function and quality of life in patients with ankylosing spondylitis after 5 years in a clinical trial of adalimumab," Rheumatology, vol. 54, no. 7, pp. 1210-1219, 2015.

[16] D. Poddubnyy, A. Fedorova, J. Listing et al., "Physical function and spinal mobility remain stable despite radiographic spinal progression in patients with ankylosing spondylitis treated with TNF- $\alpha$ inhibitors for up to 10 years," The Journal of Rheumatology, vol. 43, no. 12, pp. 2142-2148, 2016.

[17] R. Westhovens and L. Annemans, "Costs of drugs for treatment of rheumatic diseases," Rheumatic \& Musculosketal Diseases, vol. 2, no. 2, Article ID e000259, 2016.

[18] R. S. Hinman, P. McCrory, and M. Pirotta, "Efficacy of acupuncture for chronic knee pain: protocol for a randomised controlled trial using a Zelen design," BMC Complementary and Alternative Medicine, vol. 12, p. 161, 2012.

[19] J. Leem, H. Kim, and H. G. Jo, "Efficacy and safety of thread embedding acupuncture combined with conventional acupuncturefor chronic low back pain: a study protocol for a randomized, controlled, assessor-blinded, multicenter clinical trial," Medicine (Baltimore), vol. 97, no. 21, Article ID e10790, 2018.

[20] B. Curda, M. Luxl, P. Glauninger, and A. Falkenbach, "Häufigkeit der Anwendung konventioneller und unkonventioneller Therapiemassnahmen bei Patienten mit Morbus Bechterew," Complementary Medicine Research, vol. 7, no. 2, pp. 85-88, 2000.

[21] Y. J. Ren, F. Li, and J. Yan, "Clinical study of du-moxibustion combined with needling huatuo Jiaji acupoint in the treatment of AS of kidney-yang deficiency," Journal of Clinical Acupuncture and Moxibustion, vol. 35, no. 6, pp. 41-44, 2019.

[22] H. L. Jia, K. Shen, and Y. C. Zhang, "Clinical observations on the treatment of 40 ankylosing spondylitis patients by combination of acupuncture and medicine," Shanghai Journal of Acupuncture And Moxibustion, vol. 24, no. 8, pp. 23-25, 2005.

[23] J. Jia, J. P. Wu, and J. Li, "Acupuncture combined with western medicine in the treatment of 30 cases of cervical type of ankylosing spondylitis," Shaanxi Journal of Traditional Chinese Medicine, vol. 26, no. 2, pp. 157-158, 2005.

[24] P. Li, Y. J. Wang, and J. G. Tian, "Meta analysis of the randomized controlled trial of acupuncture for ankylosing spondylitis," Rehabilitation Medicine, vol. 29, no. 3, pp. 66-72, 2019.

[25] W. J. Zhao, W. Liu, and Y. H. Wu, "Meta analysis of clinical randomized controlled trials research literature on acupuncture and moxibustion treatment of ankylosing spondylitis," Journal of Basic Chinese Medicine, vol. 24, no. 12, pp. 1743-1749, 2018.

[26] X. Zhang, X. Z. Luo, and Q. Y. Cao, "Efficacy of acupuncture and sulfasalazine for ankylosing spondylitis: a network metaanalysis," Chinese Archives of Traditional Chinese Medicine, vol. 36, no. 10, pp. 2321-2324, 2018.

[27] L. Gan and X. C. Zeng, "A meta-analysis of the therapeutic effect of acupuncture and moxibustion on ankylosing spondylitis," Rheumatism and Arthritis, vol. 4, no. 9, pp. 32-34, 2015.

[28] J. P. T. Higgins and S. Green, Cochrane Handbook for Systematic Reviews of Interventions Version 5.1.0, The Cochrane Collaboration, London, UK, 2011.

[29] R. X. Yin, Y. Chen, and Y. Cai, "Effect of internal thermal needle on pain and spinal motor function in patients with ankylosing spondylitis," Journal of Hubei University of Chinese Medicine, vol. 20, no. 6, pp. 71-73, 2018.

[30] X. Chen, S. R. Chen, and S. Y. Shi, "Clinical effect on ankylosing spondylitis with electroneedling at Jiaji acupoint," Journal of Yunnan University of Traditional Chinese Medicine, vol. 40, no. 6, pp. 89-91, 2017.

[31] Y. J. Wang and W. J. Qiu, "Observations on the efficacy of superficial needling therapy for early ankylosing spondylitis," Shanghai Journal of Acupuncture and Moxibustion, vol. 36, no. 9, pp. 1088-1091, 2017.

[32] J. L. Dong, Y. B. Li, and Y. Cai, "Observations on the therapeutic effect of electrothermal needling on ankylosing spondylitis and its impact on IL-1 and IL-6," Shanghai Journal of Acupuncture and Moxibustion, vol. 36, no. 4, pp. 444-448, 2017.

[33] J. L. Zhao, "Observation on the effect of needling points on improving the disease activity and functional activities of patients with ankylosing spondylitis," Shanxi Journal of Traditional Chinese Medicine, vol. 37, no. 7, pp. 907-908, 2016.

[34] H. L. Zhang and J. Y. Zhang, "Study on the effects of needling on Jiaji acupoints and Dumai acupoints on the spinal function activity and pain of patients with ankylosing spondylitis," Medical Recapitulate, vol. 21, no. 7, pp. 1314-1316, 2015. 
[35] Y. J. Wang and W. J. Qiu, "Clinical observation of treating 20 cases of ankylosing spondylitis with superifcial needling," Rheumatism and Arthritis, vol. 3, no. 7, pp. 28-36, 2014.

[36] S. H. Wei, "Clinical observation of the treatment of ankylosing spondylitis with fire needling," Medical Frontier, vol. 2, no. 26, pp. 343-344, 2012.

[37] J. Y. Zhang and H. L. Zhang, "Clinical observation on treatment of ankylosing spondylitis with needling at Huatuo Jiaji points and Dumai points," Hebei Journal of Traditional Chinese Medicine, vol. 29, no. 7, pp. 624-625, 2007.

[38] Z. W. Zhang, J. L. Wang, and Z. W. Su, "Therapeutic effect of fire needling on ankylosing spondylitis," Liaoning Journal of Traditional Chinese Medicine, vol. 32, no. 8, pp. 822-823, 2005.

[39] Z.-T. Lv, X. Zhou, and A.-M. Chen, "Akupunktur versus krankheitsmodifizierende Antirheumatika in der Behandlung der ankylosierenden Spondylitis-eine Metaanalyse," Complementary Medicine Research, vol. 22, no. 6, pp. 395-402, 2015.

[40] H. C. Lai, Y. W. Lin, and C. L. Hsieh, "Acupuncture-analgesiamediated alleviation of central sensitization," Evidence-Based Complementary and Alternative Medicine, vol. 2019, Article ID 6173412, 13 pages, 2019.

[41] S. G. Yu, X. H. Jing, and Y. Tang, "Acupuncture and moxibustion and immunity: the actuality and future," Acupuncture Research, vol. 43, no. 12, pp. 747-753, 2018.

[42] Q. Liu, "Clinical study on treatment of ankylosing spondylitis with adjusting kidney by acupuncture and long snake moxibustion," M.S. thesis, Guangxi University of Chinese Medicine, Guangxi, China, 2018.

[43] W. B. Zeng, L. Luo, and W. C. Li, "Clinical study of filiform fire-needling Back-Shu points in the treatment of ankylosing spondylitis," Journal of Clinical Acupuncture and Moxibustion, vol. 34, no. 12, pp. 40-42, 2018.

[44] B. Xue and W. Liu, "Clinical curative effect evaluation of acupuncture on ankylosing spondylitis," China Journal of Traditional Chinese Medicine and Pharmacy, vol. 31, no. 6, pp. 2328-2330, 2016.

[45] J. L. Zhang, X. D. Liu, and Y. Chen, "Effect of bee acupuncture on TNF- $\alpha$ and IL- $1 \beta$ in peripheral blood of ankylosing spondylitis," Zhejiang Journal of Traditional Chinese Medicine, vol. 45, no. 2, pp. 136-137, 2010.

[46] G. V. Glass, "Primary, secondary, and meta-analysis of research," Educational Researcher, vol. 5, no. 10, pp. 3-8, 1976.

[47] S. Dong, J. Dai, and Y. T. Chen, "A survey of the clinical application needs of clinical doctors in the tertiary hospitals in Guangzhou for evidence-based medicine and meta-analysis," Journal of Clinical Medical Literature, vol. 5, no. 28, pp. 170-171, 2018. 